\title{
Classification of Dermoscopy Images for Early Detection of Skin Cancer - A Review
}

\author{
Ebrahim Mohammed Senan \\ PhD Research Scholar \\ Department of Computer Science, Dr. Babasaheb \\ Ambedkar Marathwada \\ University, Aurangabad, India
}

\author{
Mukti E. Jadhav, PhD \\ Marathwada Institute of Technology, \\ Aurangabad, India
}

\begin{abstract}
Early diagnosis of skin cancer is essential health requirement for the patient and a critical task for the dermatologist. The factual thinking is that the chance of patient's survival is high if diagnosed early. Analysis of the skin images and dermoscopy is a mandatory for medical professionals to take appropriate decision on treatment. A number of methods have been researched to use automated and computerized system for skin diseases image processing. Various dermoscopy image processing techniques have been reviewed to explore the possible solution to skin diseases and to select an appropriate method for early detection 7 of skin diseases. This review work will be a pathway to scientist, research scholars and medical practitioners.
\end{abstract}

\section{Keywords}

Dermoscopy, Skin cancer, Feature extraction, Classification.

\section{INTRODUCTION}

Computer modelling and image based health diagnosis is a method widely practiced by the doctors and health specialists. These models are vital in diagnosis, cure and treatment planning, apart from the biological investigation. It is considered as a modern health care practice accepted by the doctors in most of the countries. Human skin forms the cover layer of the body forming part of the integumentary system the largest organ. The seven layer of the skin which includes ectodermal tissues guarding the muscles, bones, ligaments and other internal organs. Human skin is constitution of mesodermal cells with melanin pigmentation which can possibly absorb dangerous UV radiation from sun light. Malignant melanoma is the most vigorous skin cancer, capable of spreading to other organs thereby increasing the death rate. Early deduction of the same is essential for successfully treating the disease. Skin cancer is found to spread to millions to the tune of 132000 identified to have melanoma. Throughout time, several clinical rules devised by dermatologists have been established in order to classify the skin lesions. The three common rules are the $\mathrm{ABCD}$ rule, the7-point list, and the Menzies rule. The ABCD rule consists of four features: Asymmetry, Border irregularity, Colour variation, Diameter and Evolving. The 7-point check list is based on seven dermatoscopic features such as atypical pigment network, blue-whitish veil, atypical vascular pattern, irregular streaks, irregular pigmentation, irregular dots/globules and regression structures. The first three features are called major criteria while the other four are minor criteria. Instead, the Menzies rule relies on the definitions of negative features (e.g., uniform colour pattern distribution) and positive features (e.g., multiple brown dots, multiple colours and broadened network[1].

\section{RELATED LITERATURE}

This review of related works explains the key elements of eachworktherebyhighlightingthemainfactorscontributingtothat specificre earch.The work of Sheha, M. A.(2012) analysed the texture by means of automatic way of detecting the melanoma skin cancer. The results of the work using automated method of classification on dermoscopy image. Feature extraction was done by GLCM and neural network was done by classification. Training and testing of the system was done using MLP classifier that were expressed in two techniques namely MLP- Automatic and Traditional[2].

Tool for annotating support for segmenting the images manually was by Ferreira, P. M. (2012). This tool was useful in presenting a easy and quick way having practical database for automatic segmentation and classification. Author took the suggestions of the dermatologist. The approach adopted uploading and displaying of image, segmenting manually, labelling regionally, reshaping the boundary, boundary edition at the posteriori stage, multi-user practical annotation and segmentation comparison and storing of segmented image[3].

In this modern study, by Nasir, M., et al. Proposed system of strategy for the detection of lesions. Evaluation of the proposed study on the HP2 database, pre-processing by DullRazor method for hair removal and unwanted pixels. In this approach the process of segmentation was done by using two ways, uniform distribution method and an active contour method, then by fusion between them for determining boundry clearly. The features are extracted based on color, texture and HOG. The features of each method are determined in the vector of features and fusion use of the three vectors, then feature selection to select the most important features and put them into a vector of features, which is as input to a classifier SVM to classify the lesion as malignant or benign. Findings obtained from the proposed system are specificity $96.7 \%$, sensitivity $97.7 \%$, F-score $97.5 \%$, and accuracy $97.5 \%$ [4].

Deep Neural Network was used for the dermatologist level in classifying skin cancer by Andre Esteval (2017), The achievement of $\mathrm{CNN}$ is $72.1+/-0.9 \%$ (mean+/- s.d.) total accuracy While the images were diagnosed by two doctors of dermatology and the accuracy of $66.0 \%, 65.56 \%$. The CNN accuracy was similar to the dermatologist details. It is observed the trained $\mathrm{CNN}$ when used on finer disease partition does better performance than the one trained in three or nine classes. This demonstrates the effective performance of partition algorithm[5].

Abbas, Q.(2012) studied orientation and segmentation of dermoscopy images. Contrast enhancement was done using a perceptual method of orientation. melanoma border detection was automatically done, this being the initial and crucial task. This novel approach of perceptual orientation was by combination of regions and segmentation using edge- based 
Technique. This is four stage system for colour contrasting and for segmentation. The RGB image obtained from dermoscopy is changed to CIE $\mathrm{L}^{*} \mathrm{a} * \mathrm{~b}^{*}$ colour space. Enhancement of lesion contrast is done by the adjustment and mapping the value of intensity of the lesion pixels. The Region of Interest $(\mathrm{RoI})$ is detected by using the hill climbing algorithm in oriented color space using the color channels $(\mathrm{L}$ $*, a *, b *)$. The final step would be to apply a adaptive method of thresholding to determine the optimal lesion border[6].

Ferreira, P. M. (2013) utilized computer aided diagnosis for the purpose of segmentation of the skin lesions of images obtained from dermoscopy. The methodology utilized for this was thresholding it automatically, using K- means, shifting by mean, growth regionally and Gradient Vector Flow(GVF)

and watershed. Uniqueness of the metric was a successful in segmenting the database of 50 images and it was conclusive that segmenting in the best way was by GVF snake method [7].

Silva, C. S.(2013) classified cutaneous lesion using colour based dermoscopy. The standard steps previously mentioned (i) Segmenting the lesion (ii) extracting and selecting the feature and (iii) classify the lesion - are not dependent on each other. As they are part of a processing chain, the errors from each processing step are brought forward and thus propagated. Authors proposed secondary approach by using non-standard dermoscopy image analysis. The proposal takes the route of selecting features, classifying images and then is the validating process[8].

In the presentation of Mendonça, T(2014) the dermoscopy images $\mathrm{PH} 2$. The database was an inclusion of lesion segmented medically, diagnosis done in the clinic with dermoscopy criteria like asymmetry, colour and differential structures which may be typical and atypical [9].

Deep learning for classifying skin lesion was an attempt made by Yap, J (2017). Image features were obtained using a modified version of ResNet-50 architecture. The output have only a flattened layer averaging pool layer of 2048 dimensional feature vector. This is referred as image feature extraction network. Late fusion performance is chosen with the support of embedded network with composition of two set of 1024 completely connected dimensional layer with activation function of ReLU with 5 way layer of softmax. This way leveraging data from different modality is performed. Three model of classification is analysed and compared, namely full multimodality classification, partial multimodality classification, single image classification[10].

Jaworek-Korjakowska, J (2014) researched on border irregularity detection. The details of the border were subjected to translation in a form of a function having appropriate peaks which will indicate the border irregularity. A four step algorithm was planned for implementing. The first step is computing a bounding box segmenting lesion of the skin. The second step is identifying the pixels of the boundary located on the lines which would connect the midpoint of the mass to the vertices. The third step is about calculating the distance from the border porting to the edge of the image. Smoothing of the signal is done using Gaussian filter. The filter smoothness the output and the deriving function is used to locate the maximum point of the function[11].

In this work presented, by Dash, S., et al(2018) use the Bilateral filter to extract the features by combining the traditional mask method with the features of Bilateral filter, which leads to high accuracy in the classification. The texture features of several parameters are extracted and used as an input to the KNN classifier. In the proposed method, the fusion method was used to merge the extracted features from the previous steps into the features vector. The method achieved varying proportions in mean filter, absolute mean filter and standard deviation filter by utilizing VisTex, Brodatz, ALOT and STex databases[12].

Ruela, M (2014) proposed a CAD coded differently for the diagnosis of skin lesions in dermoscopy imaging. The uniqueness of this program is there is evident importance given to the symmetry and shape features applicable to the detection of melanomas. Also commonly used are based on ABCD rule ie Asymmetry, Border, Colour and Differential features. This helps in the evaluation of each type of features. $\mathrm{PH} 2$ data base was used for testing the annotated image. The proposed system of CAD achieved sensitivity of 96\% ,specificity of $86 \%$, utilizing symmetry features and sensitivity of $83 \%$, specificity of $78 \%$ with shape features. [13].

Barhoumi, W (2014) detected the pigment network in dermoscopy images to diagnose melanoma. The key working in the method is using fuzzy logic for hole selection. This would eventually illustrate the nodes of pigment network with the support of Gaussian membership function , which filters only the holes pertaining to pigment network and simultaneously remove other circular structures ( globules, brown dots, white cysts and oil bubbles,). This is allowing a model which is accurate in identifying the uncertain decision in removing holes. This scheme reduction in pigment network holes' wrong classification. This falls under the ROC curve which is of 0.821 for doing correct distinguishing of lesions without and with pigment network[14].

This paper was presented by Aswani VS (2017) to detect skin cancer. The ABCD rule has been used to extract the features of dermoscopy $\mathrm{PH} 2$ images; in this approach two basic components were taken to analyse the skin lesion at the real time. To detect and prevent skin lesion. First, Real-time alert to prevent sunburn. Second, Analyse the image by using many steps as: improving and removing unwanted components using the median filter. Then segmentation the image to separate the area of the lesion from the rest of the image and select the region of interest. The $\mathrm{ABCD}$ rule to extract features from the region of interest(RoI)[15]. Afifi, A (2017) designed a system of efficiency in diagnosingmelanomaindermoscopy Imaging. Author used a comprehensive data sets taken from the area of tumor which was representing $\mathrm{ABCD}$ clinical rule. The feature was filtered using thetechnique of eliminating recursive features and utilizing random forests algorithm. Only important features were utilized. Extra tree classifier was build to get ensembling of classifiers utilizing various feature subsets and got accurate results[16].

Bag-of-Features method of classifying dermoscopy images is only popular approach in dermscopy. This was investigated by Chakraborty, S (2017). Here a concept of global and local attributes were adopted by the author. Global attributes was for initial assessment of lesion in the skin and local attributes also addressed as dermoscopy alphabet, were from various local patches like globulus, streaks, structure in pigmentation. A method adopted here was Gray Level Co-occurance Matrix(GLCM). The matrix gives several statistical data for computing the construction of feature vector like contrast, correlation, homogeneity, energy and entropy [17]. 
Oliveira, R. B (2017) used combined features to study on the computing the diagnosis of skin lesions. They used four spaces of colour which includes RGB, HSV, CIE Lab and CIE Luv. These were for extracting of colour and properties of the texture. For the purpose of evaluation of this method, six different classifiers were adopted namely $\mathrm{kNN}$, Bayes Networks, C4.5 decision tree, MLP, SVM and OPF. The evaluation of the performance of these classifiers were by using correlation coefficient, GRFS, gain on information, relief-F, PCA and CFS[18]

In this work done by Nezhadian, F. K (2017) in his work ISIC_UDA-2_1 with 60 images taken up for evaluating 23 for benign and 37 of malignant melanomas. Plotting of Histopathologies against relevant analyser scores were done. The average scores of all melanoma groups were found to be more than the average scores of benign PSL groups[19].

In this work by Catarina Barata (2013) used BoF bag of feature model classifying the melanomas with two variety of descriptors namely texture and colour. It is a classification system medically inspired for melanoma identification problem which is a simplified mechanism describing different dermoscopy features Barata, C., et al came out with a detection system with three blocks of processing namely 1 ) pre-processing 2) Network enhancement 3) Network Detection. During the pre-processing artifacts are detected and are removed. In the second stage the network structure is worked out taking into consideration the colour along with spatial properties and at the third stage the pigment presence is detected with the help of available information. This algorithm was run with 55 dermoscopy images with 12 images of pigment network[20].

M.E.Celebi et al worked on the diagnosis of boundary of lesion in skin cancer by using dermoscopy through

JSEG algorithm. Colour and texture were extracted with the help of GLCM and classification suggested by SVM was used to diagnose malignancy of lesions. 655 dermoscopy images were used as database for implementation and the results demonstrated an accuracy fo $90 \%$ [21].

In this paper a method was presented by Okuboyejo, Damilola A., et al(2013). to classify dermatology. The images are enhanced by DullRazor technology and median filtering to remove unwanted pixels. The method of Fuzzy sets to segment the image and determine the lesion area from the healthy skin. ABCD rule to extract features from RoI. Based on feature vector, the image is classified as malignant or benign through the known multi-layer Perceptron (MLP)[22].

In this paper, a method is presented by Abbas, Qaisar, et al (2013). Lesiton detects the boundary lesion. 100 images of dermoscopy are used for analytical purposes. Normalized Smoothing Filter (NSF) executed by average smoothing filter for the purpose of improving images and removing bubbles and hair. Local entropy, mixture models technique and global thresholding for extract features from the area lesion. The results of the True Positive Rate (TPR) are $92.10 \%$, the False Positive Rate (FPR) is $6.41 \%$ and the values (EP) 5\%,[23].

In this study, Oliveira, Roberta B,. et al (2016). presented a mathematical method for detecting skin lesions, for 480 images. The images are enhanced through an anisotropic propagation filter. The isolated lesion from the healthy skin is determined by the Chan-Viz model. The feature are extracted from the lesion area using the ABCD rule. Support Vector Machine (SVM) technique for the classification of lesion as malignant or benign . Results reached 79.01\% [24]
In this study, by Kasmi, Reda,. et al.(2016). method of classification of skin lesions in the EDRA database. The images were enhanced by gabor filters and median filter to remove all unwanted effects. The technique of active contours to separate the area of the lesion from the rest of the image. Features are extracted from the area of the lesion by ABCD rule. The results obtained from proposed system are sensitivity of $91.25 \%$, specificity of $95.83 \%$ and accuracy of 94\% [25].

In this study, Barata, Catarina, et al.(2014). Experimented two systems to detect skin cancer using the texture feature and features of the color, for a database consisting of 176 Dermoscopy images. Lesion are isolated from the rest of the image using the threshold algorithm. ABCD rule is used to extract the feature of the color from the lesion and the graylevel cooccurrence matrix (GLCM) to extract the

In this paper, Abuzaghleh, Omar,. et al.(2015). presented a system for real-time skin lesion analysis to detect and prevent skin cancer. All images of the $\mathrm{PH} 2$ database enhanced through the 84 direction filter set, region of interest is determined by the active contour algorithm and Otsu's method.The feature in the vectors are classified to identify the malignant or benign lesions by SVM technique. The result reached accuracy of melanoma, benign and atypical images are $94.3 \%, 93.5 \%$ and $90.4 \%$ respectively [27]

In this study, Nasr-Esfahani, Ebrahim, et al. (2016). presented a method for analyzing clinical images of skin cancer using convolutional neural network. The use of methods to enhance the quality of images, the technique k-means to identify similar samples in clusters, ABCD rule applied to extract the features in the database images at the University Medical Center Groningen (UMCG), CNN technique for classification of images as melanoma or benign. The result obtained from the proposed system is Accuracy of $81 \%$, Sensitivity of $81 \%$ and Specificity of $80 \%$ [28].

In this study, presented by Cavalcanti, P. G., et al.(2013). Suggested a method using the standard camera to distinguish skin lesions, pre-processing using the replacing Vorig by Vproc and Shading attenuation algorithm. The segmentation using the Otsu threshold method and thresholding based algorithm to separate the lesion area from healthy skin. Extraction of features through $\mathrm{ABCD}$ rule, classification techniques of lesion are by using the maximum Likelihood and kNN (K-Nearest Neighbors). Result obtained from classification techniques is $99 \%$ of accuracy and $100 \%$ of sensitivity [29].

In this study, by Celebi, M. Emre,. et al.(2014). presented a method of classification of skin lesions using significant colors in lesion. There are many algorithms to extract colors directly or indirectly as a standard for diagnosis. In this study the use of machine learning to extract important colors from clinical images, $\mathrm{K}$ - means is used to reduce the number of colors to $\mathrm{K}$ of colors. Through a regression algorithm is used to classify a set of 617 images as benign or malignant. Results achieved on the proposed system a specificity is $76 \%$ and a sensitivity is $62 \%$ [30].

In this study, by Barata, C., et al. (2014). Proposed new color detection system in dermoscopy images. Using Gaussian mixtures to learn a statistical model for eachcolor. The system showed the best results achieved when combining the La*b* and HSV color spaces, and the system works well where the average correlation of Superman 0.7981[31]. 
In this study, by Goel, R., et al (2015). Proposed system for analyzing features and technique of classification of skin cancer. segmentation the skin lesion from the rest image using the K-Means clustering, which depends on the color features of the cluster. Extract the features from the region of interest using GLCM algorithm by texture the image of inside the lesion. Calculate the extracted features as Correlation, Entropy, Energy, Correlation and Homogeneity. Use the classifier SVM based on the features of GLCM to classify images as benign or malignant[32].

In this study, by Riaz, Farhan, et al.(2018). proposed system for the detection of skin cancer. In this system, the boundaries of the lesion appear irregular, such as streaks and pigment networks instead of natural spots that have regular boundaries, to segmentation the lesion and separate the region of interest from the rest of the image using an active contour method followed by difference Kullback Leibler between the skin and lesion, features are extracted by Local Binary Patterns(LBP) that take the main features of skin cancer. In the proposed system, results achieved on the two database $\mathrm{PH} 2$ and ISIC, reach the accuracy with $\mathrm{KNN} 87.7 \%$ and with SVM $90.45 \%[33]$.

In this study, by Abbas, Q., et al.(2013). The system for the detection of lesions based on the colors in the lesion depends on the Menzies approach. Evaluation of the separation of the color class using transformed divergence separability and Jeffries-Matusita distances, which led to the improvement of the system and the determination of color as the features of lesion. The proposed system achieved $90 \%$ accuracy on the 120 image[34].

In this paper, by Jaisakthi, S. M., et al.(2018). Skin lesion method using the GrabCut and kmeans algorithms. The system consists of two stages: pre- processing to eliminate unwanted noise and pixels using filter techniques. Segmentation for separating of the lesion from healthy skin is done by using the GrabCut algorithm. The second phase uses the $\mathrm{k}$ - mean clustering algorithm with colors features in the lesion to improve the boundary. Results obtained from the proposed system Dice coefficient values of 0.9139 for PH2 and 0.8236 for ISIC 2017[35].

In this paper, by Suryapraba, M., et al (2015). The proposed system includes Wavelet Transformation for image enhancement and noise reduction. Extraction of features from the region of interest, the features of texture extracted by (GLCM) technique, and the puts of features in the vector features are used as the inputsin the classification techniques known Artificial Neural Network (ANN) for classification of lesion as benign or malignant, which achieved the accuracy of $95 \%$ [36].

In this work presented, by Al-Masni, M. A., et al. The use of the deep full resolution convolutional for skin lesion segmentation in dermoscopy images. In this proposed, each individual pixel is recognized without the need for preprocessing or pos-processing processes. Experience has shown the generation of spatial resolution features. The proposed system was used in the databases ISCI 2017 and $\mathrm{PH} 2$, Results obtained from the FrCN method for segmente the skin lesions is better than FCN, U-Net, and SegNet methods [37].

Diagnosing melanoma using SVM having only useful image features was the work of Mustafa, S (2018). RBF kernel is the first hyper parameters having $C$ for cost parameter and $Y$ for kernel parameter. $\mathrm{Y}$ gets divided into two sets one is the set of training using $70 \%$ data(140 images) and second set is testing set using $30 \%$ of the data(60 images). S(i,j) calculation was done with 15 features extraction. This being the condition, exhaustive grid search was done to determine the optimum turning parameters $\mathrm{C}$ and $\mathrm{Y}$ that would be required for improving SVM-RBF performance[38].

This paper suggests by Ramteke, N. S (2013). to automated analysis and detection of skin cancer. In the area of the lesion, the proposal is used to segment the lesion and determine the boundaries by using the watersheds to separate the skin cancer from the healthy skin; and to enhancement the images using pre-processing methodes. The ABCD rule distinguish was present to extract the most important features that distinguish the images as benign or malignant. The diagnostic system assessed by a database of 30 cancer images and non-cancer images, that achieved a 90\% accuracy[39].

Moura, N (2018) had done experiment with texture, color and shape descriptors as well as CNN and was applied to skin lesion image. They applied $\mathrm{ABCD}$ rule for extracte of features. GLCM was used as texture descriptor with division of features for GLCM[40].

Highest level of accuracy can be achieved in the detection of melanoma and melanocytic nerves using deep learning for two step classification for classification of malignant pigmented skin lesions, with second deep learning model giving better results. This was examined by Kaymak, S (2018). The first model $78 \%$ accuracy, the second model $84 \%$ accuracy and the third model 58\%[41].

A double stage model of segmentation and classification was built by Eric Vander Putten (2018).FCRN which has the ability to identify lesion inan image and eliminate information which are extraneous. 152 layered depth was used for classification and segmentation. It was worked on ResNets , a novel neural network (NN) model to build hundreds of residual block which were utilized in the work[42].

Detection of acral melanoma was detected by $\mathrm{Yu}, \mathrm{C}(2018)$ using convolutional neural network. VGG-16 Model having 38 million parameters was used as state of the art CNN. As small data sets are not suitable for CNN, this is overcome by fine tuning technique which is one of the technique of regularization. Modified VGG model having 16 layers has been developed using convolution filter having the size (ie 3 $\mathrm{x} 3$ ) for all convolution layers[43].

CNN results gives a the accurate value of $80.23 \%$ and $83.51 \%$ which was higher than the non-expert's evaluation $(62.71 \%$, $67.84 \%)$ and close to that of the expert $(81.64 \%, 81.08 \%)$. The area under curve values exhibit values 0.84 and 0.8 and Youden's index of 0.6073 and 0.6795 having similarity with the scores of expert. Using machine learning, dermoscopy was done on[44].

In this paper presented by Navarro, F., et al(2019). The method of gementation of the lesion in two different ways to the same image from the ISIC 2017 database, which produces high accuracy in the classification [45].

Natural image classification is one of the recent area of research performed by Harangi B. (2016) with several CNN architectures. They utilized technology like GoogLeNet ,AlexNet, ResNet, VGGNet etc.It is further possible to use the pre-processing models and extract the weights and biases. Appropriate fine tuning of all the layers used in this model can be typically applied for classification. There are few more architectures AlexNet and VGGNet in which initialization is 
done where the weights and biases are not influenced by visual examination, which may be different from the skin images [46]

Monisha, M (2017) worked on classifying malignant melanoma having benign skin lesion with the support of back propagative neural network and $\mathrm{ABCD}$ rule. The steps followed for achieving a distinctive advancement in the malignant melanoma are acquiring the image, pre-processing, highlighting the character of skin features. The extraction of features are done in an featured image processing way by incorporating ready strategy, recognising the symmetry and border, shades and discovering the dimension[47].

This paper of Pedro Rebouças Filho (2018) presented a novel method of classifying melanoma in an automatic fashion with the support of structural co-occurrence matrix (SCM) having main frequency extracted fromdermoscopy imaging. Transforming SCM in an adaptive feature extraction helps in improvement of discriminating power having image as the only parameter. This results. A comparative study with LBP showed that SCM gave better results. Results showed frequency range for SCM was working automatically, with better results compared to local binary patterns with the same data sets. The results of the proposed system were: Spe $99.4 \%, 95.23 \%$ and $92.15 \%$,Sen $99.2 \%, 94.57 \%$ and $89.9 \%$, AUC 99\%,92\% and 90\% in PH2,ISIC 2016 and2017 respectively [48].

Patiño, D (2018) worked on super pixel merging to segment skin lesion automatically. It was achieved by using SLIC algorithm for over segmenting and subsequently merging the output super pixels into two zones namely healthy skin and lesion. The average RGB colour of minutesuperpized was used as merging criterion. This methodology has overcome issues of dermoscopy like hair removal, bubbles of oil, illumination change and reflected image with no additional stage of working. PH2 and ISIC 2017 dataset have been evaluated in this method[49]

In the work done by Aljanabi, M (2018) artificial bee colony algorithm was used for lesion segmentation. A binomial image was generated for each image and then it was compared with grouth truth(GT) to get the computational value of: accuracy (ACC), sensitivity (SEN), specificity (SPE), dice coefficient (DIC), and Jaccard index (JAC). Accuracy demonstrated the relationship of segmentation analysis with overall pixel[50].

Geodesic active contours for lesion segmentation was proposed Vasconcelos, F. F. X (2018). The method is superior to the traditional method as it has the capability of adapting to the contours of the lesion using mathematical calculations and morphology. This helps in evolving the geodesic active contour and also for inserting the gradient of morphology which would delimit the lesion edges. MGAC also gave a presentation of better performance in vital metrics as compared to other techniques especially with reference to the time of performance. This is done in shorter span of time[51].

\section{CONCLUSION}

In this paper various researches done on classification and early detection of skin cancer in dermoscopy images have been reviewed. Computer based image processing and its development in the field of clinical dermoscopy has been explained in detail. Various techniques in pre-processing, segmentation, feature extraction and classification process have been reviewed and appropriately explained. This review work is expected to be a helping tool for researchers, medical practitioners and scientist.

This review is helpful in coming out with future scope of work in the field of medical imaging and processing.

\section{REFERENCES}

[1] Pomponiu, V., Nejati, H., \& Cheung, N. M. (2016, September). Deepmole: Deep neural networks for skin mole lesion classification. In 2016 IEEE International Conference on Image Processing (ICIP) (pp. 2623-2627). IEEE.

[2] Sheha, M. A., Mabrouk, M. S., \& Sharawy, A. (2012). Automatic detection of melanoma skin cancer using texture analysis. International Journal of Computer Applications, 42(20), 22-26

[3] Ferreira, P. M., Mendonça, T., Rozeira, J., \& Rocha, P. (2012, May). An annotation tool for dermoscopy image segmentation. In Proceedings of the 1st International Workshop on Visual Interfaces for Ground Truth Collection in Computer Vision Applications (p. 5). ACM.

[4] Nasir, M., Attique Khan, M., Sharif, M., Lali, I. U., Saba, T., \& Iqbal, T. (2018). An improved strategy for skin lesion detection and classification using uniform segmentation and feature selection based approach Microscopy research and technique, 81(6), 528-543.

[5] Andre Esteval et al (2017) Dermatologist-level classification of skin cancer with deep neural networks doi:10.1038/nature21056

[6] Abbas, Q., Garcia, I. F., Emre Celebi, M., Ahmad, W., \& Mushtaq, Q. (2013). A perceptually oriented method for contrast enhancement and segmentation of dermoscopyimages. SkinResearchand Technology, 19(1), e490-e497.

[7] Ferreira, P. M., Mendonça, T., \& Rocha, P. (2013, June). A wide spread of algorithms for automatic segmentation of dermoscopy images. In Iberian Conference on Pattern Recognition and Image Analysis (pp. 592-599). Springer, Berlin, Heidelberg.

[8] Silva, C. S., \& Marcal, A. R. (2013). Colour-based dermoscopy classification of cutaneous lesions: an alternative approach. Computer Methods in Biomechanics and Biomedical Engineering: Imaging \& Visualization, 1(4), 211-224.

[9] Mendonça, T., Ferreira, P. M., Marques, J. S., Marcal, A. R., \& Rozeira, J. (2013, July). PH 2-A dermoscopy image database for research and benchmarking. In 2013 35th annual international conference of the IEEE engineering in medicine and biology society (EMBC) (pp. 5437-5440). IEEE.

[10] Yap, J., Yolland, W., \& Tschandl, P. (2018). Multimodal skin lesion classification using deep learning. Experimental dermatology, 27(11), 1261-1267.

[11] Jaworek-Korjakowska，J., \& Tadeusiewicz，R. (2015, August). Determination of border irregularity in dermoscopy color images of pigmented skin lesions. In Conference proceedings:... Annual InternationalConference of the IEEE Engineering in Medicine and Biology Society. IEEE Engineering in Medicine and Biology Society. Annual Conference (Vol. 2015, pp. 2665-2668) 
[12] Dash, S., Senapati, M. R., \& Jena, U. R. (2018). K- NN based automated reasoning using bilateral filter based texture descriptor for computing texture classification. Egyptian Informatics Journal, 19(2), 133-144..

[13] Ruela, M., Barata, C., Marques, J. S., \& Rozeira, J. (2017). A system for the detection of melanomas in dermoscopy images using shape and symmetry features. Computer Methods in Biomechanics and Biomedical Engineering: Imaging \& Visualization, 5(2), 127-137.

[14] Barhoumi, W., \& Baâzaoui, A. (2014). Pigment network detection in dermatoscopic images for melanoma diagnosis. Irbm, 35(3), 128-138.

[15] Aswani VS., Hema S. (2017). ADVANCED MELANOMA DETECTION USING ABCD RULE. ISSN (PRINT): 2393-8374, (ONLINE): 2394-0697, VOLUME-4,ISSUE-12, 2017

[16] Afifi, A., \& Amin, K. M. (2017, December). An efficient system for Melanoma diagnosis in dermoscopy images. In 2017 12th International Conference on Computer Engineering and Systems (ICCES) (pp. 58-65). IEEE.

[17] Chakraborty, S., Mali, K., Banerjee, S., Roy, K., Saha, D., Chatterjee, S., ... \& Majumder, S. (2017, November). Bag-of-features based classification of dermoscopy images. In 2017 4th International Conference on OptoElectronics and Applied Optics (Optronix) (pp. 1-6). IEEE.

[18] Oliveira, R. B., Pereira, A. S., \& Tavares, J. M. R. (2018). Computational diagnosis of skin lesions from dermoscopy images using combined features. Neural Computing and Applications, 1-21.

[19] Nezhadian, F. K., \& Rashidi, S. (2017, October). Melanoma skin cancer detection using color and new texture features. In 2017 Artificial Intelligence and Signal Processing Conference (AISP) (pp. 1-5). IEEE.

[20] Barata, C., Marques, J. S., \& Mendonça, T. (2013, June). Bag-of-features classification model for the diagnose of melanoma in dermoscopy images using color and texture descriptors. In International Conference Image Analysis and Recognition (pp. 547-555). Springer, Berlin, Heidelberg.

[21] M.E. Celebi, el al. "A methodological approach to the classification of dermoscopy images," Computerized Medical Imaging and Graphics, vol. 31. no. 6, pp. 362373, 2006.

[22] Okuboyejo, Damilola A., Oludayo O. Olugbara, and Solomon A. Odunaike. "Automating skin disease diagnosis using image classification." Proceedings of theWorld Congress on Engineering and Computer Science. Vol. 2. 2013.

[23] Abbas, Qaisar, et al. "Unified approach for lesion border detection based on mixture modeling and local entropy thresholding." Skin Research and Technology 19.3 (2013): 314-319.

[24] Oliveira, Roberta B., et al. "A computational approach for detecting pigmented skin lesions in macroscopic images." Expert Systems with Applications 61 (2016): 53-63.

[25] Kasmi, Reda, and Karim Mokrani. "Classification of malignant melanoma and benign skin lesions: implementation of automatic ABCD rule." IET Image Processing 10.6 (2016): 448-455.

[26] Barata, Catarina, et al. "Two systems for the detection of melanomas in dermoscopy images using texture and color features." IEEE Systems Journal 8.3 (2014): 965979.

[27] Abuzaghleh, Omar, Buket D. Barkana, and Miad Faezipour. "Noninvasive real-time automated skin lesion analysis system for melanoma early detection and prevention." IEEE journal of translational engineering in health and medicine 3 (2015): 1-12.

[28] Nasr-Esfahani, Ebrahim, et al. "Melanoma detection by analysis of clinical images using convolutional neural network." Engineering in Medicine and Biology Society (EMBC), 2016 IEEE 38th Annual International Conference of the. IEEE, 2016.

[29] Cavalcanti, P. G., Scharcanski, J., \& Baranoski, G.V. (2013). A two-stage approach for discriminating melanocytic skin lesions using standard cameras. Expert Systems with Applications, 40(10), 4054-4064.

[30] Celebi, M. Emre, and Azaria Zornberg. "Automated quantification of clinically significant colors in dermoscopy images and its application to skin lesion classification." IEEE systems journal 8.3 (2014): 980984.

[31] Barata, C., Figueiredo, M. A., Celebi, M. E., \& Marques, J. S. (2014, May). Color identification in dermoscopy images using gaussian mixture models. In 2014 IEEE International Conference on Acoustics, Speech and Signal Processing (ICASSP) (pp. 3611-3615). IEEE

[32] Goel, R., \& Singh, S. (2015). Skin cancer detection using $\mathrm{glcm}$ matrix analysis and back propagation neural network classifier. International Journal of Computer Applications, 112(9)

[33] Riaz, Farhan, et al. "Active Contours Based Segmentation and Lesion Periphery Analysis For Characterization of Skin Lesions in Dermoscopy Images." IEEE Journal of Biomedical and Health Informatics (2018)

[34] Abbas, Q., Emre Celebi, M., Garcia, I. F., \& Ahmad, W. (2013). Melanoma recognition framework based on expert definition of ABCD for dermoscopy images. Skin Research and Technology, 19(1), e93-e102.

[35] Jaisakthi, S. M., Mirunalini, P., \& Aravindan, C. (2018). Automated skin lesion segmentation of dermoscopy images using GrabCut and k-means algorithms. IET Computer Vision, 12(8), 1088-1095.

[36] Suryapraba, M., Rajanarayanee, G., Kumari, P., \& Santhi, P. (2015). Analysis of Skin Cancer Classification Using GLCM Based On Feature Extraction in Artificial Neural Network. International Journal of Emerging Technology in Computer Science \& Electronics, 13(4).

[37] Al-Masni, M. A., Al-antari, M. A., Choi, M. T., Han, S. M., \& Kim, T. S. (2018). Skin lesion segmentation in dermoscopy images via deep full resolution convolutional networks. Computer methods and programs in biomedicine, 162, 221-231.

[38] Mustafa, S., \& Kimura, A. (2018, January). A SVMbased diagnosis of melanoma using only useful image 
features. In 2018 International Workshop on Advanced Image Technology (IWAIT) (pp. 1-4). IEEE.

[39] Ramteke, N. S., \& Jain, S. V. (2013). ABCD rule based automatic computer-aided skin cancer detection using MATLAB. International Journal of Computer Technology and Applications, 4(4), 691.

[40] Moura, N., Veras, R., Aires, K., Machado, V., Silva, R., Araújo, F., \& Claro, M. (2018, June). Combining ABCD Rule, Texture Features and Transfer Learning in Automatic Diagnosis of Melanoma. In 2018 IEEE Symposium on Computers and Communications (ISCC) (pp. 00508-00513). IEEE

[41] Kaymak, S., Esmaili, P., \& Serener, A. (2018, November). Deep Learning for Two-Step Classification of Malignant Pigmented Skin Lesions. In 2018 14th Symposium on Neural Networks and Applications (NEUREL) (pp. 1-6). IEEE.

[42] Eric Vander Putten(2018) Deep Residual Neural Networks for Automated Basal Cell CarcinomaDetection 2018 IEEE EMBS International Conference on Biomedical \& Health Informatics (BHI)4-7 March 2018.

[43] Yu, C., Yang, S., Kim, W., Jung, J., Chung, K. Y., Lee, S. W., \& Oh, B. (2018). Acral melanoma detection using a convolutional neural network for dermoscopy images. PloS one, 13(3), e0193321.

[44] S.Kalaiarasi et al, International Journal of Computer Science and Mobile Applications, Vol.6 Issue. 4, April2018, pg. 109-118

[45] Navarro, F., Escudero-Viñolo, M., \& Bescós, J. (2019).
Accurate segmentation and registration of skin lesion images to evaluate lesion change. IEEE journal of biomedical and health informatics, 23(2), 501-508.

[46] Harangi, B. (2018). Skin lesion classification with ensembles of deep convolutional neural networks. Journal of biomedical informatics, 86, 25-32.

[47] Monisha, M., Suresh, A., Bapu, B. T., \& Rashmi,M. R. (2018). Classification of malignant melanoma and benign skin lesion by using back propagation neural network and $\mathrm{ABCD}$ rule. Cluster Computing, 1-11.

[48] Rebouças Filho, P. P., Peixoto, S. A., da Nóbrega,R. V. M., Hemanth, D. J., Medeiros, A. G., Sangaiah, A. K., \& de Albuquerque, V. H. C. (2018). Automatic histologicallycloserclassificationofskinlesions.Computeri zed Medical Imaging and Graphics, 68, 40-54.

[49] Patiño, D., Avendaño, J., \& Branch, J. W. (2018, September). Automatic skin lesion segmentation on dermoscopy images by the means of superpixel merging. In International Conference on Medical Image Computing and Computer-Assisted Intervention (pp. 728-736). Springer, Cham.

[50] Aljanabi, M., Özok, Y., Rahebi, J., \& Abdullah, A. (2018). Skin Lesion Segmentation Method for Dermoscopy Images Using Artificial Bee Colony Algorithm. Symmetry, 10(8), 347

[51] Vasconcelos, F. F. X., Medeiros, A. G., Peixoto, S. A., \& Rebouças Filho, P. P. (2019). Automatic skin lesions segmentation based on a new morphological approach via geodesic active contour. Cognitive Systems Research, 55, 44-59. 